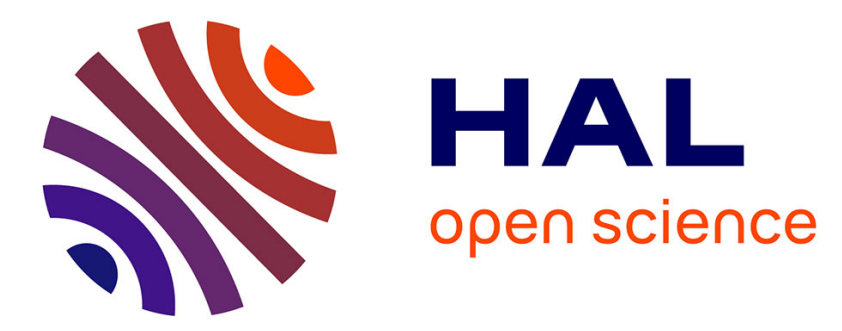

\title{
Applied Ideality: From Concepts to Inventive Problem Solving Algorithm
}

Evgeniy E. Smirnov

\section{To cite this version:}

Evgeniy E. Smirnov. Applied Ideality: From Concepts to Inventive Problem Solving Algorithm. 19th International TRIZ Future Conference (TFC), Oct 2019, Marrakesh, Morocco. pp.45-54, 10.1007/9783-030-32497-1_5. hal-02905545

\section{HAL Id: hal-02905545 \\ https://hal.inria.fr/hal-02905545}

Submitted on 23 Jul 2020

HAL is a multi-disciplinary open access archive for the deposit and dissemination of scientific research documents, whether they are published or not. The documents may come from teaching and research institutions in France or abroad, or from public or private research centers.
L'archive ouverte pluridisciplinaire HAL, est destinée au dépôt et à la diffusion de documents scientifiques de niveau recherche, publiés ou non, émanant des établissements d'enseignement et de recherche français ou étrangers, des laboratoires publics ou privés. 


\title{
Applied Ideality: from Concepts to Inventive Problem Solving Algorithm
}

\author{
Evgeniy E Smirnov \\ St. Petersburg International Public University of TRIZ, Saint Petersburg, Russia \\ triz-onemail.ru
}

\begin{abstract}
The paper is devoted to the concept of an ideality and its derivatives: an ideal functional system and an ideal outcome. The differences between them and the scope of their practical application are studied.

The current state of art in modeling and problem solving algorithms in TRIZ are also highlighted.

A simple analysis-synthesis algorithm for inventive problem solving is proposed using the concept of an ideal outcome and a resource approach aimed to reducing the ideality of the result through a resource analysis of the situation. The proposed algorithm, in contrast to ARIZ, was created to solve problems with a single conflict, i.e, without related (conjugated) functions that form a contradiction of conditions. In addition to the concepts mentioned above, the algorithm relies on the "solution backwards" method and the use of elementfunctional modeling of conflicts (EFM.C).

The paper also provides an example of analysis-synthesis for a simple production problem in order to show the mechanics of individual steps of the algorithm, as well as the entire algorithm as a whole.
\end{abstract}

Keywords: Ideality, Ideal Functional System, Ideal Outcome, Ideal Final Result, Resource Analysis, Analysis-Synthesis Algorithm, ARIZ.

\section{Introduction}

The subjects of the study are concepts and toolkit of the science of creativity and its heuristic part - contemporary TRIZ, sharpened to solve non-standard (inventive) problems. There are many disagreements on various terms and concepts. In particular, this concerns one of the key concepts of TRIZ - ideality and its applications to the result and functional system.

At this point there are two main disadvantages in the interpretation of the stated subjects: (1) such concepts as value and ideality are placed on the same shelf; (2) the concepts of an ideal system and an ideal outcome are mixed.

There is also no simple tool for working with these concepts: for analyzing the conflict model and further synthesis of solution ideas.

All existing algorithms can be divided into two categories. The first one includes overly complex tools that contain almost all concepts - from conflicts and contradictions to resources and physical effects - for example, the algorithm of inventive prob- 
lem solving (ARIZ). The second one includes tools that are weak in terms of heuristics and dialectics, and they are not effective enough in solving complex extraordinary problems - for example, such sets of techniques as SIT, ASIT and so on.

For these reasons, it makes sense to deal with the aforementioned concepts and with the basic tool of contemporary TRIZ to increase the effectiveness of their application.

\section{Background}

In contemporary TRIZ, conceptual modeling is used. This implies precise definition of all the concepts used. To this end, the formula of concepts definition has been proposed [1].

The purpose of this study is to understand the ideality, its derivatives (an ideal functional system and an ideal outcome) and the development of practices for their application.

Historically, the ideal outcome in TRIZ is called the "ideal final result" (IFR). Such a name most likely implies a final solution that suits the inventor. But it has significant disadvantages. First, ideality itself is already a limiting abstraction. Secondly, the result finiteness creates a sort of psychological barrier which limits the search for possible alternative solutions to the problem.

It should be noted that IFR was not immediately introduced into the first versions of ARIZ, although it is analogous to an element of the well-known method of solution backwards, which was proposed by Pappus (III-IV centuries A.D.), a Greek mathematician and founder of Heuristics. In modern times this method was revived by George Polya (1945) [2].

However IFR is often used independently due to the fact that it has high heuristic power. And since "ideality" is the key concept here, it often happens that IFR is confused with another similar concept - the "ideal system".

For instance, the work [3, p.76] states that the ideal final result can be achieved by the application of different tools through use of resources, and also that systems become simpler, not more complex, because: Ideality $=\Sigma$ Benefits/( $\Sigma$ Costs $+\Sigma$ Harm $)$. Although evolution is always a development which is the equivalent of the increasing complexity.

A similar definition of ideality is presented in many other works, for example, in the book [4]. At that, the authors write about the ideal result. Here is another vivid example when the ideal result is defined as an ideal system: "It's when something performs its function and does not exist" [5, p.23].

On the other hand, one of the first definition of value is $[6, p .5]$ :

1. Value is always increased by decreasing costs (while, of course, maintaining performance).

2. Value is increased by increasing performance if the customer needs, wants, and is willing to pay for more performance.

Thus, it is necessary to precisely define all these concepts and figure out how to use them in practice to successfully solve inventive problems. 
Today, the only tool within the classic TRIZ developed for this purpose is the ARIZ-85C (its latest version approved by Altshuller) [7]. In case of single conflicts, either inventive techniques or so-called Substance-Field (Su-Field) Analysis [7] are usually used. Both of these tools are seriously outdated and do not have acceptable, sufficiently effective analogues.

ARIZ-85C has a mechanism for using the concept of "IFR". But the algorithm is practically not used because it is complex, contains a large number of steps and it is necessary to formulate contradictions of different types.

In this connection, it is proposed to use a simplified version of the algorithm for solving problems without revealing contradictions. This new algorithm based on the key concepts of contemporary TRIZ: desired outcome, ideal outcome, and elementfunctional resources (EFR). For this, it is necessary to clarify these concepts, and outline ways of their practical application to eliminate conflicts in the framework of solving inventive (non-standard) problems.

\section{$3 \quad$ Ideality and Outcome}

If the value tends to infinity, it turns into the ideality. In other words, the ideality is the ultimate value. Taking value as the ratio of functionality to cost, it is possible to obtain a number of ways to idealize the target object (Fig. 1).

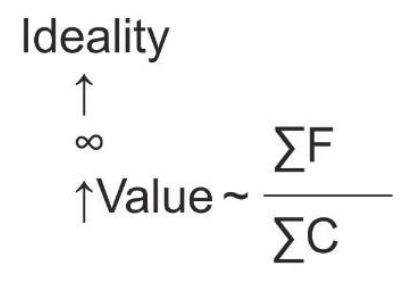

Fig. 1. Representation of ideality.

The ideality can also be achieved in another way. Since the object model is an abstraction, the maximum abstraction assumes presence of only the most significant feature - the function. This also agrees with the value formula.

Next, it is necessary to make a clear choice about what outcome one needs to obtain under the problem conditions. This outcome will depend on the type of the function involved in the conflict model. All conflicts are associated with five types of actions (Fig. 2):

1. Infliction of harm.

2. Excessive or over activity.

3. Unstable (nonpersistent) action.

4. Weak or insufficient activity.

5. No activity or malfunction. 


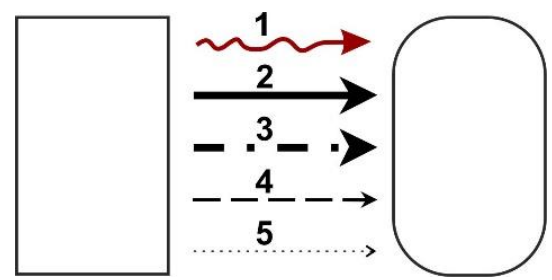

Fig. 2. Types of conflict activities.

Thus, the desired outcome will represent a new future state of the function: 1) harmful function - to eliminate or neutralize; 2) inadequate functions - to normalize; 3) missing function - to provide.

And only after the desired (or even required) outcome is determined, it is necessary to start its idealization.

\section{$4 \quad$ Idealization - for System and Outcome}

The science of creativity (or invention) and contemporary TRIZ deal with objects of reality while at the level of abstractions one develops models that allow of achieving goals and solving problems that stand in the way of these goals.

Real objects are modeled as functional systems (FS), which serve as one of the main subjects of study. The complete schematic of the functional system was presented in the author's work [1].

Modeling is the process of selecting the essential features of the object. Whereas idealization (or absolutization) assumes working with the most essential feature only, and is the highest form of abstraction. For functional systems, it is the function as a model for changing the object of function. And the outcome of the function implementation is the outcome of the change - the new state of the object of function (Fig. 3). This concept of the desired outcome is true for a useful, adequate (normal) function.

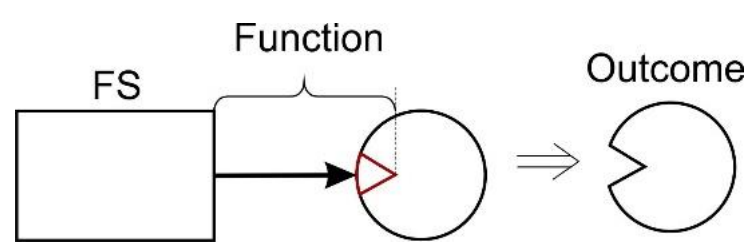

Fig. 3. Model of function and possible outcome.

It is for this reason that the ideal functional system (IFS) is reduced to a function. Cost is a relative or even subjective factor, and therefore is not taken into account in ideal models.

Thus, the IFS is formed by way of application of the idealization operator to the "functional system" concept, and has rather psychological meaning than the practical one. 
The ideal outcome is designed to be used within the framework of algorithms, but it is often used independently, which, supposedly, is the reason for the loss of its original meaning.

The algorithmic approach uses the ideal outcome concept, when the desired state of a function (an object of function) is achieved by itself, with no costs for the process of obtaining it. The ideal desired outcome (IDO) as well as the ideal functional system are the benchmarks that make it possible to use resources for implementation of the required functions.

G. Altshuller proposed another version of the IDO - for detection and measuring problems - which was recorded only in the short video of 1974: "How to search for Cinderella? Formulate the ideal final result. How to make it perfect? There is no one except Cinderella $<\ldots>$ ". For example, if it is required to find a needle in a haystack, then the IDO can be formulated in the same way - there is nothing but a needle. That is, generalizing this principle, everything that stands in the way (obstacle to the goal) should somehow disappear.

The direct interaction of the "ideal system" and "ideal outcome" concepts can occur when it is necessary to ensure the required action if there was a function missing in the original conflict. Here, ideally, the function should be implemented by the missing system that is the ideal system.

\section{$5 \quad$ Basic Analysis-Synthesis Algorithm for Conflicts Resolving}

To achieve the ideal desired outcome, a simplified algorithm based on mobilization of resources is proposed.

\subsection{Analysis}

At this stage the object under consideration is analyzed, and the environment is evaluated.

Step 1. Initial situation. Decomposition of form. It is necessary to make a list of all the elements available and their features. If the conflict is clearly localized, it is sufficient to specify only those elements which belong to the operational area.

Step 2. Revealed conflicts. Decomposition of structure and functions. It is necessary to highlight the main conflicts between the selected elements: obstacles, harm, inadequate or missing activity.

$N B$. It is possible to select all interactions between all the elements starting from the first step. But it will complicate the analysis process. If there are too many elements, then it is more convenient to switch to a purely analytical tool - for instance, value (triple) analysis. This particular algorithm is designed for the localized conflicts. Therefore, the number of such elements will be minimal. 
Also, it is necessary to indicate here the type of each function: harmful, useful (excessive, insufficient and unstable), missing (see Fig. 2).

Step 3. Desired outcomes. It should describe the final outcomes that will suit you. That is, when there is no conflict, and everything works properly: there is no obstacle, no harm, the required interactions are ensured.

If several conflicts are considered, it is necessary to describe the outcome for each activity. The desired outcomes will depend on the function type and correspond to the number of harmful, inadequate and missing functions.

Step 4. Idealization of the desired outcomes. At this step, the ideal representation of all the desired outcomes (see Step 3) for each function (see Step 2) is carried out. In other words, the ideal processes for achieving the desired outcomes should be formulated. The results are achieved by themselves (automatically), with no cost.

"Self" is the key operator that tunes thinking process to a creative mode, and removes doubts about the feasibility of achieving the desired outcomes.

The ideal outcome is only the guiding star. It is necessary try to maximize the system value by changing the functionality and cost. This provides several ways to achieve perfection. For example, it is possible to add functions to the elements imagining that these functions are performed - themselves, without additional costs and support from other elements.

\subsection{Synthesis - De-Idealization of Outcomes}

At this stage, the fabulousness wrapper is discarded, and it is necessary to proceed to the new formulations of the process of achieving the specified ideal outcomes - it is achieved, but not by itself. Although the operator "itself" may be retained for greater effect.

It is necessary to start the search for resources and their application. But, in accordance with the proposed algorithm, most of the resources have already been collected in Steps 1 and 2. So, it remains to apply them. To do this, it is required to consistently insert the available resources (see Frame 1 and Frame 2 in the template - Fig. 4) in the following wording:

$<$ Resource or its feature $>$ [itself] ensures the achievement of $<$ the (ideal) desired outcome $>$.

Step 5. Use of conflict area resources to achieve ideal outcomes. The 1st step backward from the ideal outcomes: resource mobilization in the conflict areas - see Elements and Functions from Step 2. That is, now it is a retreat from the ideal, and anything will not happen by itself (not automatically), but with the use of assistants, some X-factors. 
In this step, only those resources are used that are within the conflict operational space. That is, the interaction is normalized only at the expense of those elements which are involved in this interaction.

Each new formulation is an additional trigger for our thinking in search for new ideas. Much more paradox formulations appear. This is exactly the main objective of the algorithm.

Step 6. Use of remaining resources to achieve ideal outcomes. This is the 2 nd step back from the ideal outcomes: mobilizing resources from the environment, system and supersystem. Here, resources should be taken from Step 1 - Elements and their Features; and from Step 2 - Functions, including space-time resources. If necessary, search for resources should be repeated.

The total number of formulations here will depend on the number of resources found. The bigger number of different resources are used, the grater departure from the ideal outcome will be.

Therefore, at this and previous steps, a number of quite specific challenges come up to be solved on one's own or delegated to appropriate specialists.

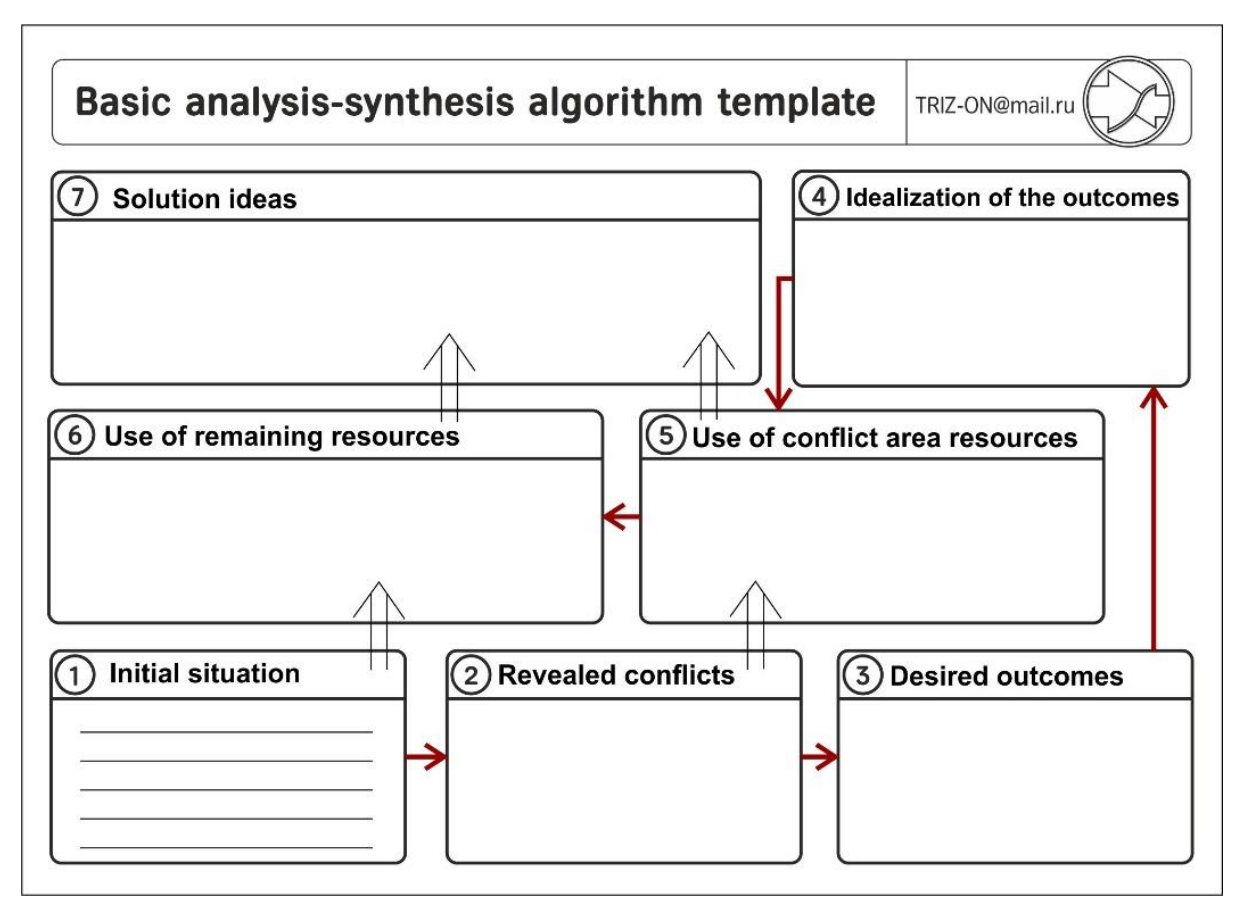

Fig. 4. Basic analysis-synthesis algorithm template.

Step 7. Solution ideas. A frame for recording solution ideas (Fig. 4). If too many ideas spring up, it is better to use a special notebook for this purpose. 
After the main resources are used - if an acceptable solution is not found or if more ideas are required - an additional step needs to be taken combining and modifying these resources (see ARIZ-85C, Part 4).

\section{How to Use the Algorithm in Practice}

In order to clarify the situation with the application of this algorithm, a simple example is offered. This will help to better understand the principles and concepts presented here.

The purpose of this example is only to demonstrate the mechanism of individual steps of the algorithm. There is no emphasis on solution ideas and their feasibility.

Part 1. Situation analysis.

Initial situation. There is a furnace for heating workpieces. To unload workpieces after heat treatment and to load new workpieces, it is necessary to open the chamber by lifting the housing. This leads to negative consequences - hot air leaves the chamber and as a result there are excessive energy consumption and time wastes for new air heating.

Step 1. Identify all available components in the situation context.

It is better that this step is performed by an expert on the system in question.

The following components are available: chamber, housing, hot air, heater elements, electric drive, workpiece, etc.

Unrecorded resources can be added later.

Step 2. Determining the area (and time) of the conflict.

The main conflict occurs in the process of reloading the workpieces - when the housing is opened, the temperature inside the chamber goes down.

What element of the chamber is specifically designed to hold hot air? Camera housing. This means that it is necessary to formulate functional conflict between the housing and hot air during the restart of the process: housing is NOT holding hot air in the workpiece processing area (Fig. 5).

In this case, in the diagram the missing (or insufficient) action is shown as a dashed line. The word form does not contain a negative particle NOT.

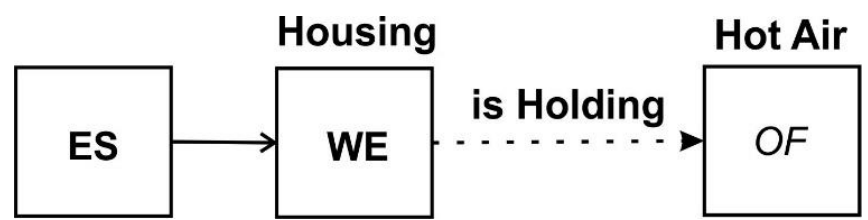

Fig. 5. Graphic representation of the element-functional model of conflict (EFM.C) [8]; where: ES - Energy Source; WE - Working Element; OF - Object of Function.

Step 3. Desired outcome formulation.

The outcome is based on the type of conflict function from Step 2. It is necessary to ensure normal (adequate) performance of the function. 
Desired outcome: housing is normally holding hot air in the workpieces processing area, that is, hot air remains within the chamber during its restart (reloading of workpieces).

Step 4. Idealization of the desired outcome.

It is necessary to formulate an ideal outcome based on how the desired outcome was set in Step 3.

Therefore, hot air itself has already remained in the chamber (during the restart).

It would be better to start this step with a mental representation. In order not to spoil the ideal image that the imagination painted, you should not write anything yet.

$N B$. This image is formulated in the perfect (completed) form. In the next steps with the use of resources, all formulations should be in the continued (process) form.

\section{Part 2. De-idealization of outcome.}

De-idealization of outcome is a synthesis of solution ideas using the resources collected during the analysis.

Step 5. Using conflicting elements as primary resources.

The formulation would be something like this: the hot air itself is holding hot air in the area during the restart process.

And here it is necessary to make another formulation - for the second element involved in the conflict (see the connection with Step 2 in Fig. 4): housing itself is holding hot air in the area inside, during the restart process.

Similar wording should also be added here for all characteristics of conflicting elements.

After that, they should be reformulated into tasks (specific challenges). It is these tasks that will need to be solved further. They are not quite inventive, but rather engineering.

For example, the task: how to make the thermal insulation layer is keeping the hot air in the process of restarting?

Such tasks should be distributed among the project participants.

Step 6. Using the remaining resources (from the system, supersystem and environment).

At this step, the resources that were taken into account at Step 1 are used.

New resources can also help other resources that are already working on achieving the ideal (desired) result from the previous step.

An example of new task: how to make cold air is keeping hot air from leaving the treatment area?

All relevant ideas should be recorded in the Frame 7: Solution ideas (see Fig. 4).

Thus, we are going from the initial situation through the inventive problem in the form of a functional conflict to engineering tasks. Some of these tasks can also be inventive problems and they must be rerun through this algorithm.

\section{Conclusions}

In the paper, the ideality idea is presented. The desired (required) and ideal outcome concepts are considered. 
The differences between the ideal outcome and the ideal functional system are shown as well.

The algorithm for solving inventive problems, based on two fundamental mechanisms of thinking - analysis and synthesis - has been proposed: thinking goes through construction of the desired outcome, its idealization, and the reverse trace (deidealization) using the rules for resource mobilization - that is, their search [analysis], choice [operational stage] and application [synthesis of solutions].

It is recommended to start learning and teaching the tools of contemporary TRIZ from this algorithm, because it is the assembly of the very first basic concepts that previously had to be studied individually: Element, Function, Functional System, Element-Functional Resources, Ideality, Ideal FS, Desired and Ideal Outcomes.

It can also be used for guided facilitation sessions for solving non-standard problems.

Also, the proposed algorithm works with single functions only. The algorithm can have at least two names, according to operating principle: Analysis-Synthesis Algorithm; and by function: Functional Conflicts Eliminating Algorithm (FCEA).

If contradictions arise, it is necessary to switch to more advanced algorithms, for example, ARIZ-85C. To work with the contradiction-removing algorithm, a preliminary study of additional concepts is needed, including: contradictions of conditions and contradictions of requirements [1].

\section{References}

1. Smirnov E.E. Formula of Concepts Definition and Its Application to Basic Concepts of Contemporary TRIZ, in TRIZ Future Conference 2018: Professional Proceedings, Strasbourg, France, 29-31 October 2018, pp. 213-223. URL: https://tfc2018.fr/professionalpapers

2. George Polya. How to Solve It. A New Aspect of Mathematical Method (1945).

3. Rantanen K., Domb E. Simplified TRIZ: New Problem Solving Applications for Engineers and Manufacturing Professionals. 2nd Edition. Auerbach Publications, 2007. - 272 p.

4. Karen Gadd. TRIZ For Engineers: Enabling Inventive Problem Solving. Oxford Creativity (2011)

5. Jack Hipple. The Ideal Result. What It Is and How to Achieve It. Springer, New York, NY, 2012. ISBN 978-1-4614-3707-9

6. Miles, L. D., Techniques of Value Analysis and Engineering (New York: McGraw-Hill, 1961).

7. Altschuller G.S., Zlotin B.L., Zusman A.V., Filatov V.I. Search for New Ideas: from Insight to Technology (Theory and Practice of Inventive Problem Solving) - Kishinev: Kartya Moldovenyaske, 1989. - 381 p. [In Russian]

8. Smirnov E.E. Element-functional modeling of conflicts: EFM.C // Three generations of TRIZ. Materials of the annual scientific and practical conference dedicated to the 90th anniversary of G.S. Altshuller, pp.4-13. - 2016. [In Russian]. URL: http://ratriz.ru/konferentsii/tri-pokoleniya-triz-2016 